\title{
SUBSTITUCIÓN DE ALIMENTO POR GALLINAZA EN LA PRODUCCIÓN COMERCIAL DE CAMARONES PENEIDOS EN HONDURAS 1
}

\author{
David R. Coddington ${ }^{2}$, Bartholomew Green ${ }^{2}$, Nahún Matamoros ${ }^{3}$, Rigoberto Rodríguez ${ }^{4}$
}

\section{COMPENDIO}

El objetivo de este investigación fue determinar los efectos de substituir alimento por gallinaza durante las primeras cuatro a ocho semanas del período de cultivo sobre la produce" de camarones peneidos y sobre la economía de producción. Doce estanques cavados en tierra (2.2-3.0 ha cada uno) ubicados en Granjas Marinas San Bernardo, S.A., San Bernardo, Choluteca, Honduras, fueron asignados a los tratamientos en forma al azar y sembrados con camarones juveniles (peso promedio $=0.8 \mathrm{~g}$ ) a razón de 5 juveniles $/ \mathrm{m}^{2}$ el 7 de septiembre de 1988. Los sistemas de manejo probados fueron: 1) Manejo estándar de Granjas Marinas San Bernardo que consiste en la aplicación de gallinaza durante las primeras ocho semanas más alimentación (ESTANDAR); 2) Alimentación solamente (ALIMENTO); 3) Gallinaza solamente durante las primeras cuatro semanas seguida por alimentación solamente (4 SEMANAS); 4) Gallinaza solamente durante las primeras ocho semanas seguida por alimentación solamente (8 SEMANAS). Los resultados, después de 99 días de cultivo, indicaron que no hubo aumento en la producción de camarones al fertilizar con bajas tasas de gallinaza (60 kg sólidos totales/ha/por semana) durante las primeras ocho semanas además de alimentar. Tampoco resultó económicamente factible substituir alimentación por bajas tasas de fertilización con gallinaza, especialmente después de las primeras dos a tres semanas de cultivo. El peso promedio de camarones en los tratamientos ALIMENTO (14.4 g) y ESTANDAR $(14.1 \mathrm{~g})$ fueron significativamente mayores que los tratamientos 4 SEMANAS (12.2 g) y 8 SEMANAS (12.1 g). La producción promedia de camarones fue de $741 \%$ mayor en los tratamientos ALIMENTO $(508 \mathrm{~kg} / \mathrm{ha})$ y ESTANDAR $(519 \mathrm{~kg} / \mathrm{ha})$ que en los de 4 SEMANAS ( $476 \mathrm{~kg} / \mathrm{ha})$ y 9 SEMANAS (368 kg/ha), sin embargo, no hubo diferencias significativas entre tratamientos, debido a la variación alta causada por la sobrevivencia variable $(\mathrm{P}<0.01)$. Los costos totales para los tratamientos ALIMENTO y ESTANDAR fueron significativamente mayores que los de 4 SEMANAS y 8 SEMANAS, debido a la mayor utilización de alimento en los tratamientos anteriores. Sin embargo, las ganancias estimadas en los tratamientos ALIMENTO (L. 3,0851ha) y ESTANDAR (L. 3,0201 ha) fueron de 27-58\% mayores que las de los tratamientos 4 SEMANAS (L. $2,389 /$ ha) y 8 SEMANAS (L. 1,9471 ha). Esto se debió a la mayor producción obtenida y los mayores precios recibidos por los camarones más grandes de los tratamientos ALIMENTO y ESTANDAR. Existió buen potencial de aumentar significativamente la ganancia estimada al substituir alimentación por fertilización con gallinaza a una mayor tasa de aplicación ( $250 \mathrm{~kg} / \mathrm{ha}$ por semana) durante las primeras cuatro a ocho semanas de cultivo.

Palabras clave adicionales: Alimentación, fertilización orgánica, Penaeus spp.

\begin{abstract}
5
The objective of this research was to determine the effects of substituting chicken manure for the common feed stuffs used In the first four to eight weeks of the growing period of shrimp. Economical evaluations of shrimp production was also considered. Twelve man made ponds (2.2-3.0 ha) located at Granjas Marinas, San Bernardo, S.A, San Bernardo, Choluteca, Honduras were assigned to four treatments at random and seeded with young shrimp (average weight $0.8 \mathrm{~g}$ ) at a rate of $5 / \mathrm{m}^{2}$ on September 7, 1998. The treatments tested were: 1) (STANDARD) the normal treatment used at Granjas Marinas San Bernardo which consisted in the application of chicken manure during the first eight weeks plus added feed, 2) (FEED) feed only, 3) (4 WEEKS) chicken manure only during the first four weeks followed by feeding, 4) (8 WEEKS) chicken manure only during the first eight weeks followed by feeding. After ninety nine days of shrimp cultivation, there was Do Increase In production using low rates of chicken manure (60 kg total solids/ha/week) during the first eight week of feeding. It was also found not to be economical to substitute feeding for low rates of fertilization with chicken manure, especially after the first two or three weeks of cultivation. The average weight of shrimp for treatments FEED 14,4, and STANDARD 14.1, was significantly greater than that observed for 4 WEEKS 12.2, and 8 WEEKS 17-1. The average shrimp production was $7-41 \%$ greater In STANDARD and FEED treatments (\%)* and $518 \mathrm{~kg} / \mathrm{ha}$ respectively) compared to 4 WEEKS $(476 \mathrm{~kg} / \mathrm{ha})$ and 8 WEEKS (368 kg/ha). However, there were no significant differences between treatments due to the high amount of variability caused by survival rates $(\mathrm{P}<0.01)$. The total costs for FEED and STANDARD were significantly greater compared to 4 and 9 WEKS. This was due to a greater feed utilization in the previous treatments. Estimated gains In FEED (L. 30851ha) and STANDARD (L. 3026/ha) were 27-58\% greater than 4 and 8 WEEKS (L. 2389/ha, and 1947/ha respectively). This was due to the Increased production obtained with greater prices received for the larger shrimp obtained in FEED and STANDARD treatments. There was potential to significantly increase the estimated gains by substituting feed for fertilization with chicken manure at a higher rate of application $(250 \mathrm{~kg} / \mathrm{ha} / \mathrm{week})$ during the first four to eight weeks of cultivation.
\end{abstract}

\footnotetext{
1 Presentado en la XXXV Reunión Anual del PCCMCA, San Pedro Sula, Honduras, 1989.

2 Department of Fisheries and Allied Aquacultures, Auburn University, AL 36849-5419 U.S.A.

3 Granjas Marinas San Bernardo S.A., Apdo. Postal 184, Choluteca, Honduras.

${ }^{4}$ Secretaría de Recursos Naturales, Dirección Agrícola Regional del Sur, Choluteca, Honduras.

5 El abatract es traducción del compendio. Elaborado por el Comité Editorial para mostrar el formato de presentación de los artículos.

Publicado en Agronomía Mesoamericana, Vol. 1 (1989).
} 


\section{INTRODUCCIÓN}

El cultivo comercial de camarones peneidos comenzó en Honduras en el año 1973, al establecerse en la costa Pacífica Sea Farms de Honduras. En 1985, otra compañía, Granjas Marinas San Bernardo S.A., construyó una finca de producción de camarones en un playón grande de la costa Pacífica. Hoy día, esta finca cuenta con 1,000 ha de estanques y está en el proceso de construir otras 1,000 ha. Un sistema de producción semi-intensiva es utilizado en Granjas Marinas San Bernardo, para así minimizar problemas relacionados con pobre calidad de agua y error humano. Generalmente se siembran juveniles de $1 \mathrm{~g}$ peso promedio, a razón de 5 juveniles $/ \mathrm{m}^{2}$ de estanque de engorde para un período de engorde de 120 días. Hay un recambio de agua diario equivalente al 5-10\% de; volumen del estanque. En caso de que la calidad de agua se deteriore es posible un recambio de mayor volumen. Alimento peletizado y bajos niveles de gallinaza son usados durante la etapa de engorde en una manera que se detalla más adelante. A pesar del éxito obvio de que goza la finca, los encargados de producción se preocupan en controlar los costos de producción, principalmente el del alimento, para poder competir mejor en el mercado mundial.

Al igual que en Panamá (Teicher-Coddington et al, en preparación), el alimento peletizado tiene un alto costo debido a que los ingredientes clave son importados. Se creyó que el alimento peletizado se podría substituir por fertilizantes orgánicos e inorgánicos más baratos y disponibles. Aunque el sistema de producción en Granjas Marinas San Bernardo incluye fertilización orgánica, la efectividad de la misma, especialmente en cuanto a reducir la necesidad de alimentar, se desconoce.

A pesar de una larga historia de uso en el cultivo de camarones, ha habido muy poca investigación sistemática en cuanto a mejorar el uso de la fertilización orgánica. En cultivos semi-intensivos estiércoles son aplicados al sembrar los camarones, seguido por aplicaciones reducidas a intervalos irregulares, dependiendo del criterio de cada productor (Trimble 1980; Pretto 1983; Garson et al, 1986). Sin embargo, los camarones también son alimentados. Recientemente, en Hawaii, U.S.A., buen crecimiento de camarones fue obtenido con el uso exclusivo de estiércol (Wyban et al, 1987). Resultados de la mayoría de los estudios, en donde sistemas tradicionales de cultivar camarones son usados, indican que el uso de alimento peletizado es necesario para obtener alta producción (Rubright et al, 1981; Garson et al, 1986; Rodríguez, 1988). Aunque alimento peletizado sea necesario además de fertilización orgánica, existe poca información en cuanto a cuando durante el período de cultivo hay que iniciar alimentación para aumentar la producción. Es posible que el mayor uso de estiércoles solamente resulte ser más económico, a pesar de ser menos productivo.
El objetivo de este ensayo fue determinar los efectos sobre la producción de camarones y economía de producción de substituir alimento por gallinaza durante las primeras cuatro a ocho semanas del período de engorde.

\section{MATERIALES Y MÉTODOS}

Doce estanques cavados en tierra (2.2-3.0 ha, cada uno) ubicados en Granjas Marinas San Bernardo S.A., San Bernardo, Choluteca, Honduras, fueron usados en este ensayo cuyo diseño experimental fue completamente al azar. Camarones juveniles (peso promedio $=$ $0.8 \mathrm{~g}$ ) fueron sembrados en todos los estanques a razón de $5 / \mathrm{m}^{2}$ el 7 de septiembre de 1988; un $10 \%$ más fueron sembrados simultáneamente para reponer la mortalidad de siembra estimada. Los juveniles procedieron de una etapa de pre-engorde posterior a su captura de los esteros. La composición de especies por estanque tuvo como promedio $89 \%$ Penaeus vannamei y $11 \%$ P. stylirostris. Los sistemas de manejo a probar fueron: 1) Manejo estándar de Granjas Marinas San Bernardo, que consiste en la aplicación de gallinaza durante las primeras ocho semanas más alimentación (ESTANDAR); 2)Alimentación solamente (ALIMENTO); 3) Gallinaza solamente durante las primeras cuatro semanas seguida por alimentación solamente (8 SEMANAS). Hubo tres réplicas por tratamiento.

La gallinaza, que consistió en aserrín de pino, estiércol, alimento desperdiciado y plumas, fue comprado en un solo lote y almacenado en sacos bajo techo hasta usarse. Se aplicó al voleo sobre la superficie del estanque a razón de $60 \mathrm{~kg}$ sólidos totales (S.T.)/ha por semana, la cual fue la práctica de rutina en Granjas Marinas San Bernardo. Los camarones fueron alimentados con ración peletizada. Durante las primeras 5.5 semanas, la ración contenía $23 \%$ de proteína, reduciéndose luego a $20 \%$ para evitar una alza en el precio. La cantidad de alimento ofrecida fue calculada según Cuadro $1 \mathrm{y}$ cantidades iguales de alimento fueron ofrecidas a camarones en estanques de un mismo tratamiento seis días por semana. La biomasa de camarones fue calculada en base a muestreos semanales y una mortalidad semanal de 0.5 y $1.0 \%$ para $P$. vannamei y $P$. stylirostris, respectivamente. Las poblaciones de camarones se muestrearon

Cuadro 1. Cuadro utilizado en calcular la cantidad diaria de alimento peletizado a ofrecerse a Penaeus vannamei y P. stylirostris sembrados en estanques a razón de 50,000 camarones/ha

(\% Biomasa de Camarones)

Peso de Camarón Penaeus vannamei Penaeus stylirostris

\begin{tabular}{lrr}
\hline$<1,6$ g/camarón & 20 & 3 \\
3,5 g/camarón & 9 & 3 \\
8,5 g/camarón & 5 & 3 \\
11,5 g/camarón & 4 & 3 \\
$>16,0$ g/camarón & 3 & 3 \\
\hline
\end{tabular}


utilizando un chinchorro de $6 \mathrm{~m}$ x $1 \mathrm{~m}$ de luz de malla de $6.4 \mathrm{~mm}$. Un total de $25-40$ camarones por estanque fueron pesados en masa por especie al 0.1 para obtener el peso promedio.

Los estanques fueron llenados por gravedad del reservorio de agua, el cual fue llenado con agua de estero. Durante los primeros 37 días se les agregó agua sólo para reponer evaporación y filtración. A partir del día 38 , hubo recambio diario de agua equivalente a $5-10 \%$ del volumen de cada estanque. El agua agregada fue filtrada a través de malla de $1.6 \mathrm{~mm}$ de luz de malla. Oxígeno disuelto y temperatura fueron medidos (medidor polarográfico de oxígeno YSI modelo 57) en todos los estanques dos veces por día entre las 2:00-4:00 a.m. y las 2:00-4:00 p.m. La salinidad del agua en cada estanque fue determinada semanalmente usando un refractómetro y una muestra de agua tomada de toda la columna de agua. La visibilidad del disco Secchi fue medida semanalmente en todos los estanques entre las 11:00 a.m. y la 1:00 p.m.

Se suspendió la alimentación el 11 de diciembre de 1988 y todos los estanques fueron cosechados el 14-15 de diciembre de 1988, después de 99 días de cultivo. El peso total de camarones con cabeza y sólo colas fue obtenido en la cosecha. Además, una muestra al azar de un promedio de 92 camarones/estanques fue sujeto a un análisis por especie del peso promedio (al $0.1 \mathrm{~g}$ ) por camarón con cabeza y sólo cola y la distribución de tamaños de cola.

Cada tratamiento fue sujeto a un análisis financiero limitado que contempló solamente los insumos principales (alimento y gallinaza) que fueron variados en el ensayo. Costos actuales incurridos y precios recibidos

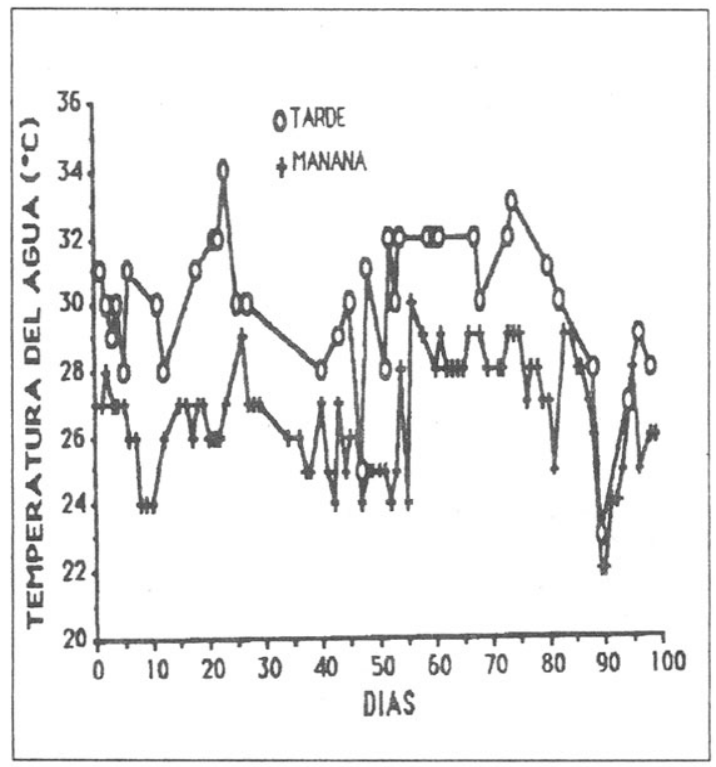

Figura 1. Temperaturas promedias $\left({ }^{\circ} \mathrm{C}\right)$ del agua por la mañana y la tarde en los estanques sembrados con camarones peneidos $\left(5 / \mathrm{m}^{2}\right)$ en Granjas Marinas San Bernardo S.A., Choluteca, Honduras. fueron utilizados. Los datos fueron analizados usando análisis de varianza con diferencias entre promedios determinadas por la prueba de rango múltiple Newman - Keuls (CLR 1987) y análisis de regresión (Feldman y Gagnon 1986). Se declararon diferencias significativas a nivel de alfa de 0.05 .

\section{RESULTADOS}

Las salinidades $(0 / 00)$ de los estanques variaron de 1 a 10 durante el período de cultivo, teniendo como promedio 3-5. Debido a las lluvias las salinidades iniciales eran bajas, sin embargo, éstas aumentaron luego del fin de las lluvias. La temperatura $\left({ }^{\circ} \mathrm{C}\right)$ del agua a tempranas horas de la mañana varió entre 22-30, con un promedio de 26.5 ; temperaturas más baja predominaron durante la primera mitad del cultivo (Figura 1).

Los promedios de oxígeno disuelto y visibilidad del disco Secchi fueron similares entre tratamientos. La concentración de oxígeno disuelto $(\mathrm{mg} / \mathrm{l})$ a tempranas horas de la mañana y la visibilidad del disco Secchi $(\mathrm{cm})$ variaron en todos los estanques entre 2.0-9.7, y 19-61, respectivamente. Los promedios ( \pm error estándar) por tratamiento durante el período del cultivo estuvieron entre $6.6 \pm 0.12$ a $6.7 \pm 0.08$ y $33 \pm 2.4$ a $35 \pm 2.1$, respectivamente. Raras veces se observaron concentraciones de oxígeno disuelto menores que $4.0 \mathrm{mg} / \mathrm{l}$ y no se observaron reacciones adversas a bajas concentraciones de oxígeno disuelto por parte de los camarones.

No hubo diferencias en crecimiento de los camarones debido a tratamiento hasta la tercera semana del cultivo, cuando los camarones en los tratamientos alimen-

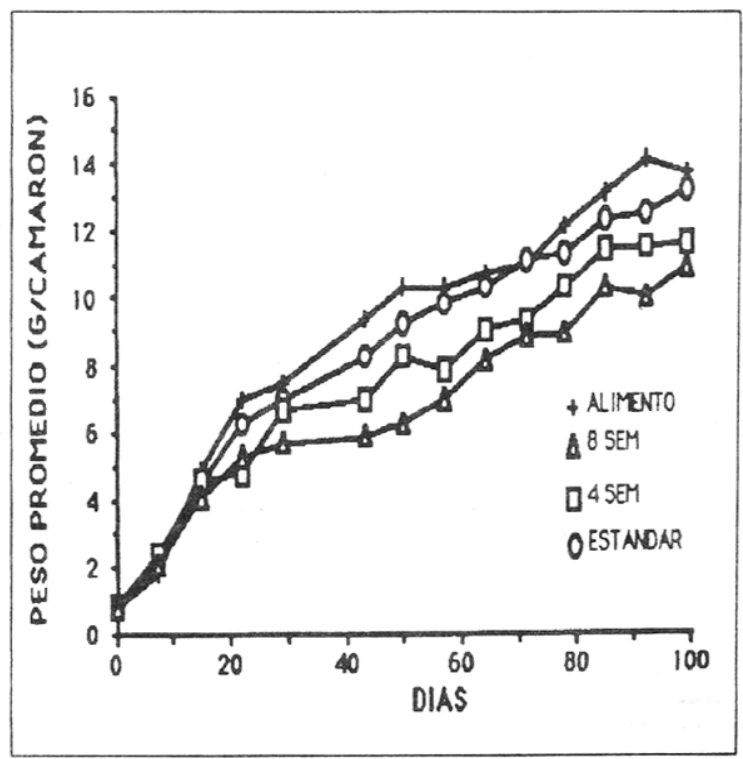

Figura 2. Crecimiento de camarones peneidos $\left(5 / \mathrm{m}^{2}\right)$ cultivados en estanques (2.2-3.0 ha) durante 99 días bajo 4 sistemas de manejo en Granjas Marinas San Bernardo S.A., Choluteca, Honduras. 
tados desde la siembra (ALIMENTO y ESTANDAR) parecían crecer más rápidamente (Figura 2). $\mathrm{Al}$ cosechar, los camarones de los tratamientos ALIMENTO y ESTANDAR fueron más grandes que los de los tratamientos 4 SEMANAS y 8 SEMANAS (Cuadro 2). Camarones más grandes resultaron en un mayor porcentaje de clasificaciones menores colas (colas/lb) (Cuadro 3). Los promedios de clasificación de cola en los tratamientos ALIMENTO y ESTANDAR fueron 17\% menores que en los de 4 SEMANAS y 8 SEMANAS.

La producción promedia en los tratamientos ALIMENTO y ESTANDAR fueron de 7 - $41 \%$ mayor que la de los tratamientos de 4 SEMANAS y 8 SEMANAS, sin embargo, no hubo diferencias significativas entre ellas debido a la alta variación dentro de algunos tratamientos (Cuadro 2). Análisis de regresión reveló que el $73 \%$ de esta variación se debió a la sobrevivencia variable $(\mathrm{P} \leq 0.01)$ (Figura 3$)$. Mayor producción tendía acompañar mayor sobrevivencia entre todos los tratamientos, especialmente en el de ALIMENTO. La cantidad total de alimento dado a los estanques explicó un adicional $17 \%$ de la variación en producción después de la sobrevivencia $(\mathrm{P} \leq 0.01)$.

\section{Economía}

Los costos totales de los tratamientos ALIMENTO y ESTANDAR no fueron diferentes, aunque si fueron mayores que los del tratamiento 4 SEMANAS, que a la

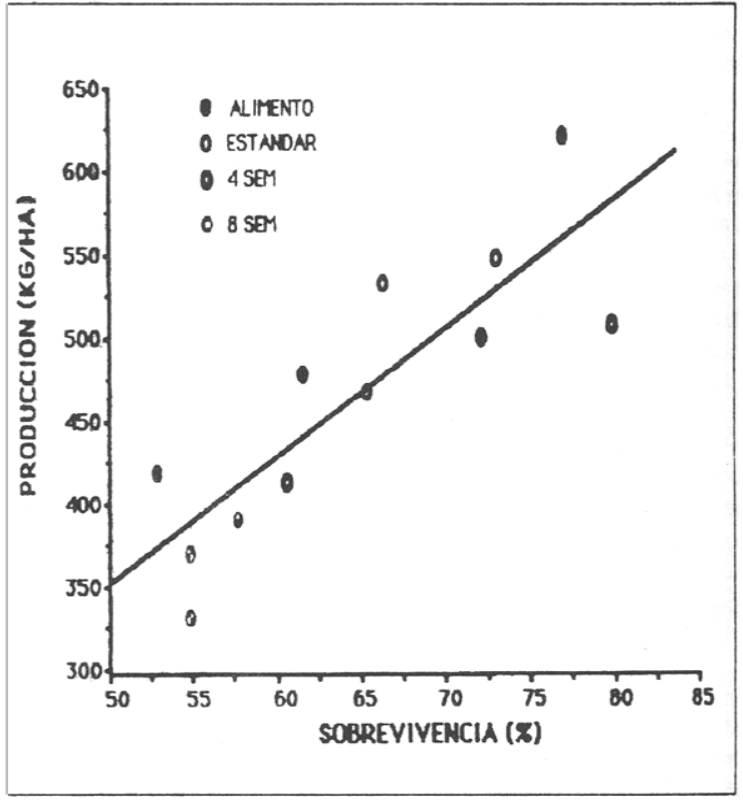

Figura 3. Relación entre producción total $(\mathrm{kg} / \mathrm{ha})$ de camarones peneidos y la sobrevivencia de los mismos. Los camarones $\left(5 / \mathrm{m}^{2}\right)$ fueron cultivados durante 99 días bajo 4 sistemas de manejo en Granjas Marinas San Bernardo. S.A., Choluteca, Honduras.

vez fueron mayores que los del tratamiento 8 SEMANAS (Cuadro 4). Subieron los costos de producción en cuanto aumentaba la cantidad de alimento usado. El costo de la gallinaza en comparación al de alimento fue

Cuadro 2. Datos de la producción (promedio \pm error estándar) de camarones peneidos (5/m2) cultivados durante 99 días en estanques (2.2-3.0 ha) bajo cuatro sistemas de manejo en Granjas Marinas San Bernardo S.A., Choluteca, Honduras

\begin{tabular}{|c|c|c|c|c|c|c|}
\hline \multirow[b]{2}{*}{ Tratamiento } & \multirow{2}{*}{$\begin{array}{c}\text { P. vannamei }{ }^{1} \\
\text { Sembrado }(\%)\end{array}$} & \multicolumn{2}{|c|}{ Peso (g/camarón) } & \multirow{2}{*}{$\begin{array}{c}\text { Producción } \\
\text { (kg/ha) }\end{array}$} & \multirow{2}{*}{$\begin{array}{c}\text { Sobrevivencia } \\
(\%)\end{array}$} & \multirow{2}{*}{$\begin{array}{c}\text { Tasa de Conversión } \\
\text { de Alimento }\end{array}$} \\
\hline & & Inicial & Final & & & \\
\hline 4 SEMANAS & $91 \pm 2$ & $0.9 \pm 0.1$ & $12.2 \pm 0.3 b$ & $476 \pm 30 \mathrm{a}$ & $72 \pm 5.8 \mathrm{a}$ & $2.0 \pm 0.1 \mathrm{a}$ \\
\hline 8 SEMANAS & $88 \pm 3$ & $0.8 \pm 0.1$ & $12.1 \pm 0.3 \mathrm{~b}$ & $368 \pm 18 \mathrm{a}$ & $56 \pm 1.0 \mathrm{a}$ & $1.5 \pm 0.1 \mathrm{~b}$ \\
\hline ALIMENTO & $90 \pm 1$ & $0.8 \pm 0.1$ & $14.4 \pm 0.1 \mathrm{a}$ & $508 \pm 60 \mathrm{a}$ & $64 \pm 7.3 \mathrm{a}$ & $2.7 \pm 0.3 \mathrm{a}$ \\
\hline ESTANDAR & $87 \pm 1$ & $0.8 \pm 0.1$ & $14.1 \pm 0.7 \mathrm{a}$ & $518 \pm 24 \mathrm{a}$ & $69 \pm 2.5 \mathrm{a}$ & $2.6 \pm 0.2 \mathrm{a}$ \\
\hline
\end{tabular}

${ }^{1}$ Otra especie sembrada fue $P$. stylirostris.

ab Promedios seguidos por la misma letra no son significativamente diferentes (nivel de alta $=0.05$ ).

Cuadro 3. Clasificación (\%)y conteo (colas/lb; promedios) de colas al cosechar camarones peneidos después de un período de cultivo de 99 días bajo cuatro sistemas de manejo en Granjas Marinas San Bernardo S. A., Choluteca, Honduras.

\begin{tabular}{|c|c|c|c|c|c|c|c|c|c|c|}
\hline \multirow[b]{2}{*}{ Tratamiento } & \multicolumn{9}{|c|}{ \% Población dentro de cada Tamaño } & \multirow[b]{2}{*}{ Conteo } \\
\hline & 21125 & 26130 & 31135 & $36 / 40$ & $\mathbf{4 1 / 5 0}$ & 51160 & 61170 & $71 / 90$ & 91/100 & \\
\hline 4 SEMANAS & 0 & 1 & 1 & 2 & 21 & 29 & 34 & 12.0 & 0 & $59 \mathrm{~b}$ \\
\hline 8 SEMANAS & 1 & 3 & 0 & 4 & 17 & 25 & 30 & 18.0 & 2 & $60 \mathrm{~b}$ \\
\hline ALIMENTO & 2 & 3 & 2 & 12 & 36 & 30 & 14 & 0.3 & 0 & $49 a$ \\
\hline ESTANDAR & 1 & 3 & 2 & 16 & 31 & 26 & 18 & 4.0 & 0 & 502 \\
\hline
\end{tabular}

ab Promedios seguidos por la misma letra no son significativamente diferentes (alfa $=0.05$ ). 
Cuadro 4. Costos de insumos e ingresos (Lempiras/ha; promedio \pm error estándar) en la producción de camarones peneidos bajo cuatro sistemas manejo en Granjas Marinas San Bernardo S.A, Choluteca, Honduras. \$1 U.S. = 2 Lempiras

\begin{tabular}{lcccccc}
\hline & \multicolumn{3}{c}{ Costos de insumos } & & \\
\cline { 2 - 4 } Tratamiento & Alimento $^{1}$ & Gallinaza $^{2}$ & Total & & $\begin{array}{c}\text { Ingreso } \\
\text { Bruto }\end{array}$ & $\begin{array}{c}\text { Ingreso } \\
\text { Neto }\end{array}$ \\
\hline 4 SEMANAS & $789 \pm 1.2$ & $16 \pm 0.0$ & $805 \pm 1.2 \mathrm{~b}$ & $3194 \pm 185 \mathrm{a}$ & $2389 \pm 186 \mathrm{a}$ \\
8 SEMANAS & $443 \pm 0.0$ & $32 \pm 0.0$ & $475 \pm 0.0 \mathrm{c}$ & $2422 \pm 163 \mathrm{~b}$ & $1947 \pm 163 \mathrm{a}$ \\
AUMENTO & $1112 \pm 0.0$ & $0 \pm 0.0$ & $1112 \pm 0.0 \mathrm{a}$ & $4197 \pm 432 \mathrm{a}$ & $3085 \pm 432 \mathrm{a}$ \\
ESTANDAR & $1080 \pm 0.0$ & $32 \pm 0.0$ & $1113 \pm 0.0 \mathrm{a}$ & $4139 \pm 440 \mathrm{a}$ & $3026 \pm 440 \mathrm{a}$ \\
\hline
\end{tabular}

1 Costo L. $0.82 / \mathrm{kg}$

2 Costo L. 0.06/kg

abc Promedios seguidos por la misma letra no son significativamente diferentes (nivel de alfa $=$ $0.05)$.

insignificante y no representó más del 7\% de los costos totales en cualquier tratamiento.

Mayores producciones y tamaños de camarones resultaron en ingresos brutos en los tratamientos ALIMENTO y ESTANDAR que fueron 29-73\% mayores que los de los demás tratamientos (Cuadro 4). Sin embargo, los ingresos netos promedios de los tratamientos ALIMENTO y ESTANDAR sólo fueron 27-58\% mayores debido a que estos tuvieron mayores costos de producción. No hubo diferencias significativas entre tratamientos debido a la alta variación en producción.

\section{DISCUSIÓN}

Estos resultados fueron concluyentes en dos aspectos: Alimento no pudo substituirse económicamente por bajos niveles de fertilización con gallinaza $(60 \mathrm{~kg}$ S.T/ha por semana), especialmente después de las primeras 2-3 semanas del cultivo; y, no hubo aumento en la producción de camarones al fertilizar con bajos niveles de gallinaza durante las primeras 8 semanas de $;$ cultivo además de alimentar. Por lo tanto, si se usa la fertilización orgánica, debería hacerse en base a cantidades suficientemente grandes para influir en la producción. En términos reales esto significa que la tasa de aplicación debe aumentarse varias veces. En cuanto a reducciones en costos de producción, la eliminación del componente de fertilización con gallinaza de manejo estándar de Granjas Marinas San Bernardo resultaría en un ahorro de L. 32/ha por ciclo. Sin embargo, debido al bajo costo de la gallinaza, es posible realizar mayores ahorros al substituir alimento por fertilización con mayores tasas de gallinaza durante las primeras cuatro a ocho semanas.

Alimento ha sido substituido con éxito por gallinaza durante las primeras semanas del período de cultivo en sistemas de agua dulce. En Honduras (Green y Alvarenga, 1989), al substituir completamente alimento por gallinaza (1000 kg S.T./ha por semana) durante las pri- meras ocho semanas o substituir parcialmente alimento por gallinaza (500 kg S.T/ha por semana) con una menor tasa de alimentación resultaron en producciones similares de tilapia a costos significativamente más bajos que en un sistema en donde se utilizó solamente alimento peletizado ( $23 \%$ proteína). Resultados parecidos se obtuvieron en Panamá, en donde el crecimiento durante las primeras cuatro semanas de un policultivo del camarón de agua dulce (Macrobrachium rosenbergii) y tilapia fue similar en los tratamientos de fertilización con gallinaza (250 kg S.T/ha por semana) y solamente alimento peletizado ( $25 \%$ proteína) (Teichert-Coddington y Peralta, 1986, datos no publicados). Es razonable esperar que un sistema similar funcionaría en estanques de producción semi-intensiva de camarones peneidos. La fertilidad natural del estanque basado en aplicaciones de carbono exógeno es adecuado para soportar biomasa pequeñas a moderadas de peces o camarones. Aplicaciones de insumos orgánicos estimulan la producción de detritus y los microbios asociados, y zooplancton (Moriarty y Pullin, 1987), los cuales son excelentes alimentos para peces jóvenes (Geiger, 1983) y camarones (Chong y Sasekurnar, 1981; Ruthbright et $a l, 1981$; Lim y Persyn, 1989). Alimento es un insumo orgánico, aunque caro si sólo actúa como fertilizante, y no es aplicado inicialmente en cantidades adecuadas para aumentar la fertilidad del estanque. Es más lógico agregar mayores cantidades de fertilizante orgánico de bajo costo, tal como gallinaza, cuando la biomasa de camarones es pequeña, y luego iniciar ALIMIENTO cuando los camarones requieran una dieta mejor para lograr su crecimiento óptimo.

La tasa de aplicación de gallinaza usada por Granjas Marinas San Bernardo S.A. fue baja, principalmente debido a temores de que tasa de aplicaciones mayores resultarían en concentraciones de oxígeno disuelto a tempranas horas del día peligrosamente bajas. Sin embargo, datos obtenidos de estanques de agua dulce sin recambio de agua en Panamá (Teichert-Coddington et 
al. 1987) y en Honduras (Green et al, 1988) indicaron que aplicaciones semanales de gallinaza de hasta $250 \mathrm{~kg}$ S.T./ha no produjeron bajas concentraciones de oxígeno disuelto ni poblaciones inestables de fitoplancton. En Hawaii (Wyban et al, 1987) alrededor de $1500 \mathrm{~kg}$ S.T. estiércol de ganado/ha por semana fueron agregados a estanques de producción de camarones, aun cuando hubo recambio diario de agua equivalente al $20 \%$ del volumen del estanque. Garson et al, 1986, aplicaron alrededor de $240 \mathrm{~kg}$ S.T estiércol de gallinaza/ha cada quince días a estanques de producción de camarones durante un período de cultivo de 120 días. Por lo tanto, recomendamos que la tasa de fertilización con gallinaza en Granjas Marinas San Bernardo S.A. sea cuadruplicada a $240 \mathrm{~kg} \mathrm{S.T} \mathrm{/} \mathrm{ha} \mathrm{por} \mathrm{semana} \mathrm{y} \mathrm{que} \mathrm{la} \mathrm{ALIMENTO}$ de las primeras cuatro a ocho semanas sea substituida por esta nueva tasa de fertilización. Resultados preliminares obtenidos de estanques de producción de 24 ha cada uno en donde se está probando el nuevo sistema de producción indican que el crecimiento de los camarones es comparable al crecimiento obtenido cuando los camarones son alimentados. Si estos resultados se mantienen habría un ahorro en gastos por concepto de ALIMENTO de L. 650.00/ha por ciclo de producción.

\section{RECONOCIMIENTO}

Agradecemos al Ing. Rubén Sánchez, encargado de producción de Granjas Marinas San Bernardo S.A.. su colaboración indispensable. Este trabajo fue traducido al Español por V.W. Green. Esta investigación fue financiada por Granjas Marinas San Bernardo S.A. y Pond Dynamics/Aquaculture Collaborative Research Support Program (C.R.S.P.), el cual es financiado por la Agencia Internacional para el Desarrollo de los Estados Unidos de Norteamérica, la Universidad de Auburn, AL, U.S.A., y la Secretaría de Recursos Naturales, Honduras.

\section{BIBLIOGRAFIA}

CHONG, V.C.; SASEKUMAR, A. 1981. Food and feeding habits of the white prawn Penaeus merquiensis. Marine Ecology - Progress Series 5:185-191.

CLR ANOVA. Analysis of variance program for the Apple Macintosh. Clear Lake Research.

FELDMAN, D.S.; GAGNON, J. 1986. Statview 511. Brain Power, Inc., Calabasas. CA, 91302, U.S.A.
GARSON, G.L; BIEBER, G.; SMITHERMAN, R.O. 19834: The effects of manures and pelleted feed on survival, growth and yield of Penaeus stylirostris and P. vannamei in Panama. Master of Science Thesis, Auburn University, AL, 36849, U.S.A.

GEIGER, J.G. 1983. A review of pond zooplankton production and fertilization for the culture of larval and finger ling striped bass. Aquaculture 35:353-369.

GREEN, B.W.; ALVARENGA, H.R; PHELPS, R.P.; ESPINOZA, J. 1988. Technical report of Cycle 3, Dry and Wet Season Phases, Comayagua, Honduras. Dept. of Fisheries and Allied Aquacultures, Auburn University, Alabama, 36849, U.S.A.

ALVARENGA, H.R. 1989. Producción de tilapia bajo sistemas de fertilización orgánica y alimentación. In Reunión Anual del PCCMCA (35, 1989, San Pedro Sula, Honduras) Compendio, de Resúmenes, San Pedro Sula, Honduras.

LIM, C.; PERYSN, A. 1999. Practical feeding-penaeid shrimp. In T. Lovell (ed.), Nutrition mid feeding of fish. Van Nostrand Reinhold. New York, U.S.A. pp. 205-217.

MORLARTY, D.J.W.; PULLIN, RS.V. (ed.) 1987. Detritus and microbial ecology in aquaculture. ICLARM. Conference Proceedings 14, International Center for Living Aquatic Resources Management, Manila, Philippines.

PRETTX), R.M. 1983. Penaeus shrimp growout in Panama. A J.P McVey, (ed.) CRC Handbook of mariculture, Vol. 1, Crustacean aquxw. ture. CRC Press, Boca Rat6rt, FL 334270, U.S.A. pp. $169-180$

RODRIGUEZ, 1. 1988. Feeding Penaeus vannamei and P. stylirostris in nursery ponds. Master of Science Thesis, Auburn University, AL 36849, U.S.A.

RUBRIGHT, J.S.; HARRELL, J.L; HOLCOMB, H.W.; PARKER, J.C. 1981, Responses of planktonic and benthic communities to fertilizer and feed applications in shrimp mariculture ponds. Journal of the World Mariculture Society 12(1):281-299.

TEICHERT-CODDINGTON, D.R., PERALTA, M.; PHELPS, R.P.; PRETTO, R. 1987. Technical report of Cycle 3, Dry and Wet Season Phases, Gualaca, Panama. Dept. of Fisheries and Allied Aquacultures, Auburn University, Alabama, U.S.A.

; ARRUE. M.; PRETTO, R.; PHELPS, R.P. In preparation. Effects d diet protein and stocking density on production of $\mathrm{Pe}$ naeus vannamei in tropical earthen ponds.

TRIMBLE, W.C. 1980. Production trials for monoculture and polyculture of white shrimp (Penaeus vannamei) or blue shrimp (P. stylirostris) with Florida pompano (Trachinotus carolinus) in Alabama, 19781979. Proc. World Maricul. Soc., 11:44-59.

WYBAN,J.A., LEE, C.S.; SATO, V.T.; SWEENEY, J.N.; RICHARDS,W, K. Jr. 1987. Effect of stocking density on shrimp growth rates in manure-fertilized ponds. Aquaculture, 61:23-32. 